\title{
Entrelacs
}

Cinéma et audiovisuel

\section{Pier Paolo Pasolini, du corps brut à la brutalité de l'image}

Fabrice Bourlez

\section{(2) OpenEdition}

Journals

Édition électronique

URL : http://journals.openedition.org/entrelacs/357

DOI : 10.4000/entrelacs.357

ISSN : 2261-5482

Éditeur

Éditions Téraèdre

Référence électronique

Fabrice Bourlez, «Pier Paolo Pasolini, du corps brut à la brutalité de l'image », Entrelacs [En ligne], 9 | 2012, mis en ligne le 25 octobre 2012, consulté le 19 avril 2019. URL : http://

journals.openedition.org/entrelacs/357; DOI : 10.4000/entrelacs.357

Ce document a été généré automatiquement le 19 avril 2019

Tous droits réservés 


\title{
Pier Paolo Pasolini, du corps brut à la brutalité de l'image
}

\author{
Fabrice Bourlez
}

\section{Le cinéma corps-à-corps}

1 Le cinéma de Pasolini est fait de corps. Il s'en dégage un érotisme singulier et un regard sur le monde à la fois sacré et profane, mythique et réaliste, désespéré et joyeux, critique et amoureux. Caméra au poing, enragé, Pasolini n'hésitait pas à jeter son propre corps dans la lutte. Film après film, le réalisateur a fait valoir les corps pour se défendre contre le néo-capitalisme triomphant, contre la petite-bourgeoisie italienne, contre tous les conformismes. Ce retour au corps, cet éclairage spécifique sur notre chair trace le fil rouge de toute son œuvre cinématographique. Face aux nouvelles déclinaisons du pouvoir - devant l'homologation des physiques et de l'esthétique, devant la répression du désir et des actions, devant la consommation outrancière, devant le moralisme bien-pensant, devant la permissivité auto-contrôlée - Pasolini fait appel au réel : contre la parole veine, la force vive des corps.

2 Que peuvent nous apprendre cet érotisme et cette rage? Comment peuvent-ils nous instruire et nous aider à comprendre la façon dont nos corps et nos gestes se modulent aujourd'hui ? Puisque le cinéma de Pasolini est habité par les corps, puisque ses images sont baignées d'un érotisme puissant, peut-il nous aider à (re)penser le genre et la sexualité ? De ce corps-à-corps cinématographique peut-il naître d'autres façons de conce voir nos désirs?

Notre hypothèse est double, elle joue sur la polysémie du mot « genre $»^{1}$.

4 D'un côté, il s'agit de comprendre comment la filmographie pasolinienne vient s'inscrire dans l'histoire du cinéma, comment elle y résiste et comment elle déjoue les mécanismes diégétiques (propres à la vision, à l'audition, à la narration cinématographique), les codifications habituelles pour s'inscrire en faux vis-à-vis de toutes classifications et brouiller les pistes des catégorisations formelles. Bref, l'œuvre de Pasolini trouble le 
genre cinématographique au point, peut-être, de le mettre à mort, d'y marquer un point d'arrêt.

De l'autre, il s'agit de comprendre comment, en raison même de ce trouble diégétique, de cette vision qui va parfois jusqu'à l'aveuglement, le regard qu'il pose sur les corps donne à penser des formes de sexualité moins stables ou moins identifiées. Son cinéma nous invite à reprendre contact avec la tension, le vacillement et la limite qui habitent nos corps. Au lieu de nous formater, l'image pasolinienne nous indique un dehors, un en-deçà de ce que nous croyons être : les bords risqués d'une nouvelle éthique?

Or le genre sert à classer les humains aussi bien que leurs œuvres. Pasolini a l'audace de faire trembler la notion ${ }^{2}$. Il se hasarde à vouloir construire un regard neuf, loin des (ar)rangements identitaires. Au-delà du genre, Pasolini regarde les corps dans leur singularité, il s'empare de leur réel pour ouvrir des voies inédites à l'écriture du cinéma et des sexes : un authentique éros cinématographique ${ }^{3}$.

7 Notre hypothèse se réfère bien sûr au style pasolinien, à son parcours, tant plastique que théorique, mais elle s'accroche aussi aux réflexions de Félix Guattari et de Teresa de Lauretis quant aux rapports qu'entretient le septième art avec le pouvoir. On serait tenté de dire que l'œuvre pasolinienne a condensé le sens de leurs développements avant même que ces deux auteurs ne les formulent dans le sillage de la pensée foucaldienne.

Teresa : «T'as de beaux yeux tu sais? », Félix : « Embrassez-moi !»

Guattari écrivait dans l'effervescence de l'après Mai 68 et de l'après Anti-〔Edipe (1971) Lauretis, italienne, immigrée aux États-Unis, prenait la plume au moment tragique des « années Sida » pour devenir l'une des principales instigatrices de la bouillonnante « queer theory ${ }^{5}$ ». Même s'ils ne semblent pas avoir eu de relation directe avec le cinéaste et même si leurs travaux paraissent à quelques années de distance et sur des continents différents, quand on les lit, on peut d'abord supposer que Guattari comme de Lauretis connaissaient l'œuvre de Pasolini sans l'avoir véritablement thématisée pour autant ${ }^{6}$. Ensuite, on peut également compter sur une certaine proximité théorique : l'un comme l'autre élaborent une pensée non académique, transversale, tendue entre pouvoir, psychanalyse et médias. Dans leurs textes s'élabore le deuil de la toute puissance du sujet moderne, coincé dans ses certitudes, englué dans sa conscience subjective, terré dans son for intérieur. Guattari comme De Lauretis privilégient des "mouvements de subjectivation", de construction par l'extérieur, par ce qui n'est pas moi. C'est dans la prise en compte de ces mouvements de subjectivation, par ces devenirs-sujets en lutte contre le pouvoir établi, que l'on pourra mieux saisir la portée de l'éros cinématographique pasolinien.

Dans un article décisif pour les réflexions sur le genre et la sexualité, Teresa de Lauretis explique que «la construction du genre se poursuit à travers des technologies de genre variées (le cinéma par exemple) et des discours institutionnels (la théorie par exemple) qui ont le pouvoir de contrôler le champ de significations sociales et donc de produire, promouvoir et "implanter" des représentations du genre $»^{7}$. Autant dire, comme Foucault nous l'avait déjà enseigné ${ }^{8}$, que ce qui pourrait sembler le plus naturel (le sexe) est irrémédiablement marqué par l'artifice et le semblant, que la sexualité est moins une affaire privée qu'un lieu déterminé par l'extériorité, que ce qui m'appartient de la façon la plus personnelle et la plus intime dépend, en fait, d'une série de normes et de conventions dont je ne suis pas propriétaire.

11 Ainsi, en quelques lignes aux accents foucaldiens - nous y reviendrons -, De Lauretis nous exproprie de toute mainmise sur notre identité sexuelle pour en faire l'objet de 
représentations sociales, sempiternellement rabâchées : «T'as de beaux yeux, tu sais ? / Embrassez-moi!».

Par combien de baisers, blancs, beaux, éclatants de propreté, hétéro-sexués, nos yeux de spectateurs occidentaux ont-ils été assujettis à la norme de ce que signifie être un homme ou (/avec) une femme? Guattari ne dit pas autre chose quand il envisage le statut du sujet comme le résultat d'un agencement de composantes hétérogènes des réalités dominantes. Il évoque justement cette domination en termes de visage. "Il existe toujours un temps, dans l'ordination de l'espace social, où la dimension de visage s'interpose pour délimiter ce qui est licite et ce qui ne l'est pas $^{9}$ ». Gageons que ce sont ces mêmes visages qui sont autorisés, ou pas, à s'embrasser à longueur de journée sur nos écrans. Au fond, pour De Lauretis comme pour Guattari, l'inconscient n'est pas strictement personnel mais renvoie à des «technologies" et à des «machines». Il y a donc un inconscient cinématographique qui déclare qu'un visage, qu'un baiser entre deux visages, que deux corps qui se touchent " "c'est comme ça", expression d'un coup de force sémiologique [...]. On identifie la "chose" ; on la localise sur diverses coordonnées abstraites ; on la tient en main, on l'empêche de fuir, de s'échapper du système des significations et de menacer l'ordre social sémiotique régnant $»^{10}$. Bref, l'image relève d'un genre donné.

La scène d'anthologie, le passage culte, le cliché, le french kiss, le baiser - gros plan, visage contre visage, bouche contre bouche, paupières fermées, lèvres peinturlurées, musique choisie -, l'inévitable spectacle nous assujettit, par sa répétition infinie, par la normalité même avec laquelle on l'identifie comme "un baiser de cinéma ». Nous sommes sujets parce que nous sommes capables de reconnaître ce que le cadre de l'écran nous montre mais l'écran nous assujettit, nous encadre, en cela justement qu'il fixe inconsciemment ce que nous reconnaîtrons comme normal. Tel est bien l'enjeu du genre cinématographique : il fixe à l'avance ce à quoi l'on peut s'attendre, le champ des visibilités. «Les thèmes du cinéma, ses modèles, ses genres, ses castes professionnelles, ses mandarins, ses vedettes sont, qu'ils le veuillent ou non, au service du pouvoir. Et pas seulement en tant qu'ils dépendent directement de la machine financière du pouvoir, mais d'abord et surtout parce qu'ils participent à l'élaboration de ses modèles subjectifs ${ }^{11}$ ".

À ce constat lucide quant au pouvoir de l'inconscient cinématographique, De Lauretis et Guattari opposent des stratégies de résistance. Elles permettront de nous rapprocher du travail de Pasolini.

\section{Le cinéma comme fantasme micro-politique}

Dans un chapitre de La révolution moléculaire, Guattari invoque un cinéma comme «art mineur » au sens où il ne corroborerait plus les représentations et les enjeux de pouvoir dominants (ou majeurs) et deviendrait une "machine de libération du désir ». Dans ce texte, Guattari remarque que même le cinéma commercial, qui constitue pourtant « une drogue adaptative $»^{12}$, n'en reste pas moins un temps durant lequel «on accepte par avance qu'il nous dépouille de notre identité, de notre passé et de notre avenir. Son miracle dérisoire, c'est de nous rendre, pour quelques instants, orphelin, célibataire, amnésique, inconscient et éternel ${ }^{13}{ }^{\prime}$. On ne pourrait mieux décrire la magie du septième art, ses puissances fictionnelles et la fascination, tout enfantine, devant le mouvement des images. 
16 fantasmatique qu'occupe le cinéma dans nos existences ; ces fantasmes fictionnels ne sont pas seulement des parenthèses dans nos existences (le cinéma comme simple divertissement) mais les déterminent inconsciemment: les fantasmes (cinématographique entre autres) encadrent en effet notre vision du monde. Certes, le spectateur décide d'aller voir un film ou pas, mais le film oriente sa manière de voir le monde. S'appuyant sur ces rouages fondamentaux, sur cette dépossession systématique qui s'impose à nous durant la séance, Guattari appelle donc de ses vœux un cinéma qui, à la place de nous conforter du côté " des visages de nos proches, clôturés sur eux-mêmes ", nous ouvrirait à un désir qui ne s'asphyxierait pas entre le " plaisir licite » et « l'interdit codifié ", un cinéma qui nous reconduirait à un réel allant au-delà de l'emprisonnement dans les représentations fantasmatiques normées, balisées. Toutefois, Guattari semble rester sur le mode programmatique, on dirait qu'il attend le changement à venir, la sortie des clichés : un fantasme micropolitique.

À l'inverse, alors que la théoricienne queer reprend la même logique que celle de Guattari pour s'intéresser aux cultures populaires, à cause de leur capacité à modeler et à couler nos êtres, elle n'attend pas des lendemains qui seraient censés chanter. Pour elle, le changement est déjà là : "Cependant, les conditions de possibilité d'une construction différente du genre existent aussi dans les marges des discours hégémoniques. Situées en dehors du contrat social hétérosexuel et inscrites dans les pratiques micropolitiques, elles peuvent contribuer à la construction du genre et elles se situent plutôt à un niveau local de résistances dans la subjectivité et l'autoreprésentation. $»^{14} C^{\prime}$ 'est notamment à cet endroit de son raisonnement qu'elle signe les fameuses possibilités de re-signification, de ré-assignation d'un nom, tellement caractéristique de la pensée queer. "Je » peut vraiment être « un autre » localement, par l'intermédiaire d'une subversion des modèles de représentation et par une auto-nomination. "Cet ailleurs n'est pas une utopie, un endroit, un temps et un espace futurs qui n'existent pas encore. Il est déjà là (...) pour déconstruire les silences de l'histoire et de nos propres constructions discursives, dans des cartographies du corps autre-ment (other-wise) érotiques et dans la mise en images et la réalisation de nouvelles formes de communauté par les sujets autre-ment désirants de cette théorie queer $»^{15}$. Le renversement du modèle dominant n'est plus à envisager comme une possibilité à venir, il est déjà à l'œuvre dans les pratiques minoritaires, dans les productions artistiques, poétiques et intellectuelles qui dé-visagent le pouvoir de la normalité au point d'en montrer à la fois l'étroitesse ridicule et la violence innommable à l'égard de ceux et celles qui ne sentent pas conformes à la norme. Pour De Lauretis, il existe des films qui ouvrent des fenêtres subversives, qui détournent le champ des visibilités et le poussent au dehors de son périmètre, actualisant ainsi d'authentiques fantasmes micro-politiques.

Bien que De Lauretis ne s'y réfère pas dans son article, il nous semble que l'œuvre de Pasolini constitue une cartographie de corps, d'images et donc de sujets «autrement désirants ", qu'elle écrit un champ de visibilités alternatives. Les contours plastiques de cette micro-politique de l'image s'imposent d'une façon tout aussi convaincante, voire plus explicite (?), que dans le cinéma de Cronenberg qui ne cesse pas de retenir les faveurs de la théoricienne queer (peut-être parce que plus populaire ?) ${ }^{16}$. Quoiqu'il en soit, une grande part de l'œuvre de Pasolini met en circulation des modèles, des représentations, une diégèse pour le moins déliée des canons hollywoodiens. Ainsi, dès les 
premières fictions, son cinéma fait-il proliférer tout un peuple mineur. Ceux qui restent généralement hors-champ entrent dans la cadre du «style indirect libre » pasolinien ${ }^{17}$.

Partons simplement des visages chez Pasolini. Leurs sourires sont parfois édentés, leurs regards noirs, profonds, leurs chevelures sombres, leurs traits fascinants d'irrégularités. Ils rient, crient ou demeurent mutiques. S'ils s'embrassent peu, ils dévisagent souvent le spectateur. Ils regardent droit dans la caméra. En cela, ils prennent le contre-pied exact de ce que Jacques Aumont remarquait si précisément : « Le visage ordinaire au cinéma est aussi celui de la démocratie occidentale, c'est-à-dire américaine et capitaliste. Il est l'un des traits de l'impérialisme, son ordinarité est un ordre ${ }^{18}$.

Que l'on se limite à songer à la manière dont les plans pasoliniens s'arrêtent sur les yeux de Silvana Mangano et Franco Citti dans EEdipe Roi (1967) aux sourires moqueurs de Ninetto Davoli et aux inimitables moues de Toto dans Des oiseaux petits et gros (1966), au bleu du regard de Terence Stamp dans Théorème (1968) ou aux véritables " gueules » des inconnus qui crèvent l'écran depuis Accattone (1961) en passant par L'évangile selon Saint Matthieu(1964) jusqu'à la Trilogie de la vie. De la beauté la plus canonique à celle des acteurs non professionnels que chérissait Pasolini, le cinéma pasolinien traite le visage en le détournant de l' " ordinarité » au point d'en faire un style immédiatement reconnaissable 19. L'étonnante singularité de ses acteurs signe son image. Qu'ils soient objectivement beaux ou laids, la caméra de Pasolini leur fait échapper à toute vulgarité que soit celle du brillant de la starification ou celle inhérente à l'absence de cultus, de soins et d'ornements.

\section{Foucault spectateur}

21 Prenons maintenant deux coordonnées plus précises. Leur repérage nous est offert par Michel Foucault lui-même. Si l'immense philosophe s'est avéré plutôt avare de commentaires sur le septième art, il se penche cependant sur deux films de Pasolini qui nous permettront de mieux cerner la façon dont son esthétique déjoue les mécanismes de normation inconsciente propre aux genre cinématographique. Autrement dit, Foucault nous permettra d'expliquer à présent comment Pasolini développe ce que l'on pourrait appeler, dans le sillon des théories de Guattari et de De Lauretis, un fantasme micropolitique.

22 À un an distance ${ }^{20}$, Salo et les cent vingt journées de Sodome (1975) et les Enquêtes sur la sexualité (1965) retiennent, tour à tour, l'attention de l'auteur de l'Histoire de la sexualité. Gageons qu'il ne s'agit pas simplement d'une coïncidence si, aux alentours de la sortie de La Volonté de Savoir, Foucault s'autorise à parler de cinéma et, en particulier, de celui de Pasolini. Il juge Salo avec sécheresse ${ }^{21}$, se réjouit des Enquêtes sur la sexualité.

Mais peu importe les goûts de Foucault. Plus que sa critique cinématographique en soi, ce qui importe ici, ce sont les effets de vérité qu'opère ce prélèvement inattendu dans l'œuvre de Pasolini. Bonne pioche en effet: quelles que soient les remarques foucaldiennes sur le contenu des films, leur rapprochement pousse à l'interprétation double, voire l'interpénétration, du cinéma avec la théorie. Qu'il les ait aimés ou pas, les films de Pasolini semblent avoir résonné au point de pousser à écrire celui qui s'efforçait, à l'époque, « de montrer comment des dispositifs de pouvoir s'articulent directement sur le corps ${ }^{22} »$. Du coup, interrogeons-nous : pris ensemble que disent ces deux films au biopouvoir $^{23}$ ? 
D'un point de vue cinématographique, ce binôme témoigne de l'évolution de la colère de Pasolini. Elle n'a cessé de croître au cours des ans, Pasolini voit son pays succomber au néo-capitalisme et à la consommation outrancière. Plus le temps passe, plus il en perçoit les stigmates sur les corps de ses contemporains. Cinématographiquement, le binôme repéré par Foucault renseigne donc sur le changement d'humeur de Pasolini, de plus en plus enragée.

\section{Des mots décors}

En 1965, Pasolini pratique l'ironie, il sourit devant les enfants, les vieilles filles et les fiersà-bras, il sillonne les routes italiennes du Nord au Sud pour réaliser un documentaire para-télévisuel ${ }^{24}$ et rendre compte des représentations de ses concitoyens en matière d'amour et de sexualité. Pareil à Socrate, il s'avance sur les routes d'Eros. Pasolini interroge, Pasolini questionne, Pasolini cherche à comprendre. Micro à la main, il se présente comme un commis voyageur qui n'a rien à vendre mais qui veut savoir. Malgré ses tentatives répétées, malgré les vains espoirs d'une re-découverte d'authenticité dans le Sud de la péninsule, malgré la diversité des accents et même, parfois, des dialectes directement sous-titrés dans la version originale, où qu'il aille, Pasolini ne dépasse pas bien le stade de la déclaration conforme, acceptable.

Ses « comices sur l'amour ${ }^{25}$ » n'accouchent que de l'opinion et du cliché. C'est ce qu'il ne cesse de déplorer dans ses entretiens avec Moravia ou Ungaretti qui scandent les différentes parties du film. Rien de très sincère n'émerge des propos que tiennent les italiens sur la sexualité. Sa tentative de "cinéma-vérité " semble vouée au ratage. L'homologation des réponses, l'impossibilité de dire quelque chose d'un tant soit peu expressif, non bridé par une pseudo-tolérance bien-pensante désespère notre auteur. Faut-il penser que la banalité des propos ne fait que taire l'embarras des interviewés ? Le langage vaudrait-il comme masque devant l'indicible du sexe ? L'option, évoquée pendant le film lors d'un bref échange avec le psychanalyste italien Cesare Musatti, est bien entendu rejetée par Foucault dans sa courte critique du film. Elle mérite quand même d'être évoquée au passage.

En ce sens, on regardera la première séquence du film, tout à fait admirable. Pasolini demande à un attroupement de jeunes garçons, qui doivent avoir entre cinq et huit ans, comment naissent les bébés. L'énigme de la sexualité et les balbutiements pour essayer d'y répondre depuis la plus tendre enfance ne pourrait être mieux illustrée. Ces enfants rient, répondent à moitié, tournent autour du pot, évoquent les fleurs ou la cigogne : ils savent sans savoir. Sans doute le film s'inquiète-t-il de cette innocence perdue ? Sans doute déplore-t-il les certitudes adultes, ce savoir si assuré avec lequel les italiens semblent maitriser leurs discours sur la sexualité. Quoiqu'il en soit, à l'époque, Pasolini s'en moque. Il censure les récits vaguement grivois, met de la musique Yéyé, sans trop se fâcher avec cette Italie dont la valse des mots pour ne pas dire, des paroles creuses, ne cessera de s'accélérer.

Bref, les propos recueillis, voulant trop faire sens, ratent ce dont l'œil de la caméra s'est, en revanche, parfaitement approprié. Car ces discours sur l'amour et la sexualité sont saisis au grand air, devant tous, dans la rue ou sur la plage, sous la lumière et la chaleur de l'été et, plan après plan, à l'image, revient ce qui passionne le cinéaste: une authentique prise sur les corps. Massés autour de l'œil de sa caméra, à moitié nus, les 
corps se collent, se serrent et frissonnent devant la difficulté de bien répondre. En-dehors du sens de leurs mots, sous les évidences faciles de leur blabla, surgit la sacralité du corps. Si on n'entend que du faux dans cet épisode de cinéma-vérité, on n'y voit que le réel des corps dont Pasolini semble éperdument amoureux. En même temps qu'elle regrette leurs idées, sa caméra enregistre leurs gestes et nous laisse - encore - accéder à l'innocence première des enfants avec lesquels le cinéaste ouvrait son film. Du coup, s'il y a à entendre quelque chose, ce ne sera que les effets de corps dans les mots: les balbutiements, les hésitations, les trébuchements de la parole, les fous rires et les hurlements qui habitent les propos policés de l'Italie interviewée. Même si, croyant trop bien savoir ce qu'ils disent, craignant ou revendiquant d'être "vieux jeu» (pensare all'antica), ses compatriotes ne témoignent que d'un conformisme accablant, la lumière qui baigne leur corps finit par séduire le regard pasolinien. Si le film traite de l'amour, c'est de celui de Pasolini pour la carnalité du sacré.

\section{Le film comme texte} enchanter, à envelopper les corps d'une troublante simplicité, à mettre à nu, à montrer comment le sacré habite la chair. En ce sens, et de façons différentes, Théorème (1968), avec une rigueur et une axiomatique proche de l'ascèse, et $L a$ Trilogie de la vie, avec joie et humour, éclaireront les puissances du corps et l'amour que leur portait Pasolini. Le corps comme lieu d'un devenir-autre, d'une pour le moins paradoxale transcendance incarnée. Le corps comme enracinement d'un érotisme libéré des conventions et d'une innocence éthique. De la sorte, le cinéma que propose Pasolini est un cinéma de la joie qui semble faire confiance aux hommes et donne à voir des déserts où vivre joyeusement à l'abri du vide de la parole.

que Pasolini finira par tourner le dos à la joie des corps en liberté qu'il avait peinte dans Le Decameron (1971), Les contes de Canterburry (1972) et Les Mille et une nuit (1974). Reste l'«abjuration ${ }^{26}$ ». Reste son dernier film : Salo ou les cent-vingt journées de Sodome (1975). Clôture violente, énigme brutale et insupportable, le film interrompt le parcours à travers les corps. Pour mémoire, rappelons que, Pasolini a choisi d'y mettre en scène le célèbre texte de Sade en le situant sous la république fasciste de Salo. Dans son roman, avec une logique aussi sulfureuse qu'implacable, le divin Marquis tentait de tout écrire sur le sexe et portait ainsi la littérature jusqu'à sa limite ${ }^{27}$. À l'écrit comme à l'écran, enfermés dans une luxueuse demeure, se succèdent les récits de mères maquerelles. Ces dernières ont été réunies par quatre nantis qui ont décidé de donner libre cours à leurs passions, de se lancer dans une débauche sans retour. Le film suit la lettre du texte mais se construit en "girons ${ }^{28}$ " qui iront de plus en plus loin dans la monstruosité, la violence et l'humiliation. Plus de trente ans après sa sortie et malgré l'omniprésence de la brutalité explicite dans les médias, le film reste un choc, un traumatisme, un impossible à regarder. Paraphrasant Blanchot, au moment où sa critique littéraire chante les louanges de Sade, il faut bien dire que " s'il y a un Enfer pour les cinémathèques, c'est pour un tel film ».

31 À la place de chanter le corps, sa transcendance ou sa sacralité, Pasolini choisit de lui infliger les pires supplices. L'association arbitraire du texte de Sade aux pratiques nazifascistes, "cette analogie grossière", pour s'exprimer comme Barthes, n'est pas à comprendre comme une réduction d'un des drames de l'humanité à une simple 
composante perverse mais, et Pasolini y est revenu à quelques occasions, comme une " allégorie ${ }^{29}$ ».

Ecoutons le réalisateur : «J'ai fait un film qui s'appelle Salo, tiré de Sade, où se voient des choses effroyables qui, en réalité, prises une à une, seraient pornographiques, vues en dehors de leur contexte ; mais, dans leur contexte, je pense qu'elles ne le sont pas, parce que le contexte est celui de la commercialisation que le pouvoir fait des corps, et alors, tous ces rapports sexuels sont une métaphore de la transformation du corps en marchandise, des corps par le pouvoir ${ }^{30} »$. Impossibilité d'utiliser les repères habituels pour classer Salo donc. Ce que l'on voit n'est pas ce que l'on croit voir. Salo va au-delà du genre. L'écriture filmique de Pasolini fait voler en éclat la distinction, tout aussi canonique que rhétorique, entre pornographie et érotisme. Il transgresse les lois du genre. Impossible de rattacher ses images à un champ de visibilités circonscrits. Paradoxalement, le texte que sa caméra donne à voir devient alors complètement inédit. On n'avait jamais vu ça.

En ce sens, la cinéaste Catherine Breillat commente le film de manière perspicace : « $\mathrm{Ce}$ que dit Salo, la littérature ne pouvait pas le dire ${ }^{31} »$. En portant Sade à l'écran, Pasolini ne trahit en rien le domaine de la littérature, mais prend en charge ce que cette dernière n'atteint pas. Plus exactement, il pousse l'écriture dans un retranchement encore plus extrême. Avec Salo, la dimension de l'image se déplace, pour faire naître une écriture filmique. En se mettant sous le signe du texte, Salo renonce à la féerie commerciale propre au spectacle du septième art. L'image choque d'abord parce qu'elle prend comme interlocuteurs les théories de Sade mais aussi celles de Klossowski, de Nietzsche, de Beauvoir ou de Sollers. Ces théories ne constituent plus des pré-textes pour le film mais des outils d'écriture pour une pensée qui échapperait aux l'illusions propres à l'image cinématographique, à l'image du baiser hollywoodien. Dans Salo, les baisers sentent la merde et le sang. Pour ceux qui font usage de Raison, le heurt fondamental devant Salo ne réside pas tant dans les risques encourus par les contenus de l'image que dans le rapport que celle-ci entretient avec le savoir des textes. «Mais il y a quelque chose encore qui n'a pas plu en son temps, qui a jeté un froid jusque chez les plus fermes partisans de Pasolini, quelque chose que l'histoire a depuis lors confirmé avec un méchant éclat : il n'est pas de pensée, de discours ou de radicalité qui ne soit intégrable par les pouvoirs modernes et récupérables pour leur propre compte. Ici, les quatre "maîtres": le Duc, l'Evêque, le Président de la cour d'Appel et le Président "philosophent" entre eux, citant à l'appui de leur folie criminelle Barthes, Klossowski, Blanchot, à propos de Sade ${ }^{32}$ ». Le jeu de Pasolini est particulièrement subtil : non seulement il entre en dialogue avec les grands lecteurs de Sade pour lui faire dire ce que son rapport à la littérature ne lui permettait pas d'affirmer mais il utilise aussi les propos de ses critiques pour montrer avec insistance comment rien ne peut s'opposer au pouvoir de l'image qui est toujours en mesure de tout récupérer et de tout détourner, notamment les grandes pensées philosophiques.

Ainsi, si la magie retorse du cinéma et le pouvoir de l'imaginaire peuvent s'accaparer toute pensée pour la détourner en son fond, la seule façon de faire dérailler l'image est de s'y installer, d'utiliser son propre pouvoir contre elle-même: la forcer à montrer "l'irregardable » afin que le spectateur sorte de son endormissement, se rebelle contre son assujettissement et réoriente son regard en fonction du réel insoutenable auquel il a été confronté. En ce sens, la célèbre " séquence des délations ${ }^{33}$ » s'avère particulièrement éblouissante. 


\section{Le film comme résistance : le cinéma mis à mort}

depuis l'entrée dans la maison ne saurait tarder. La musicienne qui accompagnait les récits des maquerelles vient de se suicider. Les jeunes victimes se mettent alors à se dénoncer les uns les autres pour sauver leur misérable existence dans la demeure des tortures. Tour à tour, ils confessent à leurs bourreaux libertins les méfaits d'autres victimes afin d'échapper à une peine qu'ils encourent. Véritable hémorragie, une révélation en entraîne une autre et les secrets renvoient, chaque fois, un peu plus loin les bourreaux dans leurs déambulations. Ils deviennent de ce fait l'objet du dire des victimes qui s'expriment pour la première fois. Enfin, celles-ci, quasi muettes depuis le début du film, sortent de leur docilité effrayante et se mettent à parler. Leurs répliques s'enchaînent: «... désobéissant à vos lois... » succède à «...quelqu'un a enfreint vos règlements ». Les libertins semblent alors glisser eux-mêmes sur la pente de la dénonciation et se rendre compte que «le véritable hors-la-loi dans Salo est la victime et non pas le bourreau ${ }^{34} »$.

Mais, plus profondément encore, ce que cette séquence dénonce c'est l'absence de résistance dans le film. On ne résiste pas dans Salo. C'est pourquoi, l'œuvre, en tant que telle, peut s'imposer comme acte de résistance. Pas de résistance dans le film mais le film comme résistance. Quitte à mettre à mort le genre artistique lui-même, quitte à aveugler le spectateur, même averti.

Ici se précise alors l'enjeu et le lien entre bio-politique et cinéma. Salo offre l'occasion d'expérimenter l'absence de résistance caractéristique du spectateur. En effet, celui qui est hors-la-loi, le premier à ne manifester aucune résistance devant Salo, comme devant les autres films, c'est le spectateur. Autrement dit, le contenu des images du film de Pasolini n'est pas insoutenable parce qu'il associe la réalité historique (le nazi-fascisme) à l'espace littéraire (les perversions sadiennes) mais parce qu'il développe un nouveau type d'écriture filmique qui dénonce la passivité dans laquelle nous plonge généralement l'image et qui suscite une réaction qui vient s'inscrire dans nos corps. Arracher Sade au domaine de l'infini renvoi des lois de la littérature, le faire passer à l'image permettrait alors d'en actualiser la dimension micro-politique. Pasolini télescope bel et bien deux cauchemars qui n'ont pas la même consistance: celui de Sade appartient à l'espace littéraire jamais clôturable tandis que celui du nazi-fascisme a irrémédiablement bouleversé la réalité du monde. Cependant, le jeu de Pasolini reste risqué et ambigu tant qu'on ne prend pas en considération l'élément premier de cette association de cauchemars : le septième art. Celui qui, par excellence, offre le spectacle censé nous faire rêver. Dès lors, Pasolini opère moins une juxtaposition de Sade au fascisme qu'une critique profonde de la violence sadique de l'image filmique. Une mise à mort de l'image.

Donc le fait que Foucault ait tracé un trait d'union entre les Enquêtes sur la sexualité, où régnait une certaine clémence quant à l'Italie et ce dernier film nous renseigne certes cinématographiquement sur l'évolution de la position de Pasolini par rapport à la société qui lui était contemporaine. Mais, au-delà de cela, le rapprochement des deux films permet également de saisir un déplacement quant au rôle de l'image cinématographique elle-même : elle n'est plus seulement censée enregistrer le réel des corps, la splendeur de leur singularité mais elle vise beaucoup plus à mobiliser le réel chez le spectateur. 

l'autre, deux couples se sont formés à l'image. Comizi d'amore s'achève par le mariage de Tonino et Graziela dont la grâce de l'amour ne veut rien savoir, inconscients de leur amour, ils répètent l'ordre hérédité de l'histoire, le geste de leurs parents, ils réitèrent l'oubli. Salo se termine par une valse hésitante entre deux jeunes garçons qui étaient au service des quatre maîtres, l'un demandant à l'autre comment s'appelle sa petite amie. Dans un cas comme dans l'autre, la scène finale pose donc la question de l'avenir, de la possibilité d'un après l'image. Cependant, alors que le final de Comizi d'amore laisse leurs illusions aux jeunes mariés, avec Salo on ne peut croire que difficilement à un dénouement heureux. Et l'ambigüité érotique de la valse entre les deux garçons laisse elle-même peu de place pour un quelconque avenir entre Marguerite, la soi disant petite amie, et le jeune homme en train de danser avec un autre garçon. Si le baiser de "fin ", occupe encore une certaine place dans les Enquêtes sur la sexualité, dix ans plus tard il s'avère totalement impossible. Il ne s'agit plus d'enregistrer la beauté de ce qui n'est plus ou risque de bientôt disparaître, il ne s'agit plus de montrer la sacralité d'un corps vivant en liberté devant l'échec de la parole contemporaine mais il s'agit alors de convoquer l'effroyable pour susciter un effet chez le spectateur : que cela s'arrête !

L'image cinématographique rentre alors encore plus pleinement dans le cadre du fanstasme micropolitique tel que nous le thématisions plus haut avec Guattari et De Lauretis. Un lien révolutionnaire se tisse dans la dernière œuvre de Pasolini entre cinéma, micropolitique et sexualité. L'image, comme une arme, vient pointer ce que Gayle Rubin, autre célèbre théoricienne de la scène queer met si justement en évidence : «le domaine de la sexualité définit des interactions politiques, des formes d'inégalités et d'oppressions qui lui sont propres. Comme c'est le cas pour les autres aspects du comportement humain, les formes institutionnelles concrètes qui régissent la sexualité, en n'importe quel lieu et à n'importe quelle époque, sont des produits de l'agir humain. Elles sont traversées par des conflits d'intérêt et des manœuvres politiques, tant délibérés qu'accidentels. En ce sens, le sexe est toujours politique ${ }^{35}$ ", même dans un simple baiser de cinéma.

\section{Défaire le genre ${ }^{36}$}

Les critiques ont souvent remarqué comment Salo désacralisait tous les rites symboliques repas, mariages, concours, noces, célébrations, ... Nous croyons que cette désacralisation vise avant tout le genre au double sens du terme, filmique et sexuel, en tant qu'opérateur et garant des visibilités et des identités. Pasolini affirmait quelque temps avant d'être cruellement assassiné : «L'idée de l'absolu privilège de la normalité est aussi naturelle que vulgaire, et franchement criminelle ${ }^{37}$ ». Il ajoutait : «Personnellement, je ne crois pas que la forme actuelle de tolérance soit réelle. Elle a été décidée "en haut" : c'est la tolérance du pouvoir de la consommation, qui a besoin d'une élasticité formelle absolue dans les "existences", pour que chacun devienne un bon consommateur. Une société sans préjugés, libre, dans laquelle les couples et les exigences sexuelles (hétérosexuelles) se multiplient, et par conséquent avide de biens de consommation ${ }^{38} »$.

Le septième art a accompagné l'essor de cette société de consommation permissive et plate. Pasolini ne vise pas le retour d'un pouvoir qui oserait explicitement affirmer son origine "d'en haut ", un ordre symbolique fort, une loi du Père qui saurait nommer les êtres. Son dernier film pousse au contraire la (dé)construction des genres jusqu'à un 
point de non-retour. Son cinéma se meut alors en écriture. À la place de rester fasciné devant la sacralité de la chair, il la raie, l'implose jusqu'à l'insoutenable. Pasolini se refuse de désigner un quelconque modèle de liberté souhaitable, il préfère abandonner toute possibilité d'assigner à l'avance le champ des visibilités : il nous aveugle. Dès lors ce qui importe beaucoup plus, c'est l'effet que la vision du film engendre sur le spectateur: le confortera-t-il dans ses habitudes de perception et de pensée ou mettra-t-il en œuvre un dispositif qui le contraindra à « revoir » ce qu'il croyait être?

Cette dernière déclinaison du fantasme micropolitique pasolinien s'avère pour le moins révolutionnaire voire, pour reprendre le mot-valise de Serge Daney, «therroriste » c'està-dire " volontiers théoricien, théorique, et pratiquant un terrorisme de la parole et de l'image ${ }^{39}$ ». De fait, Pasolini réussit à inventer une nouvelle langue, capable de prendre le contre-pied de la tolérance permissive caractéristique du capitalisme des images. Cette langue matérialise le regard, brutalise l'œil. Elle filme jusque l'impossible.

\section{NOTES}

1. Polysémie utilement éclairée par les contribution du récent recueil dirigé par J. Wasiolka, Genres en mouvement, Paris, Nouveau Monde édition, Coll. Cies Sorbonne, 2009.

2. Cf. Dans son livre sur la difficile théorie de la sexuation lacanienne, Guy Le Gaufey opère une généalogie intéressante $d u$ "genre » en tant que catégorie de pensée en mesure de classifier l'être humain lui-même. Cf. G. Le Gaufey, Le pastout de Lacan. Consistance logique, conséquences cliniques, Epel, Coll. Lacan, Paris, 2006.

3. Nous employons ici cette expression malgré la mise en garde guattarienne contre le terme «machine d'éros » qu'il aurait préféré remplacer par «machine de libération de désir ». Cf. F. Guattari, La révolution moléculaire, Fontenay-sous-bois, Recherches, coll. Encre, 1977, p. 225.

4. Sur la relation de Guattari avec Deleuze et sur leur modalité d'écriture, on doit renvoyer, avant tout, à leur immense production qui s'envisage comme un " agencement collectif d'énonciation » où l'on peut difficilement cerner ce qui revient à l'un ou à l'autre des deux auteurs. Les deux textes de référence étant bien entendu G. Deleuze - F. Guattari, Capitalisme et schizophrénie 1, AntiEEdipe, Paris, Minuit, 1971 et G. Deleuze - F. Guattari, Capitalisme et Schizophrénie 2, Mille Plateaux, Paris, Minuit, 1980. Pour une approche plus historique du rapport de Guattari à la publication Cf. F. Dosse, Gilles Deleuze et Félix Guattari. Biographie croisée, Paris, La découverte, Paris, 2007.

5. Littéralement, théorie de l'étrange, du bizarre, du pédé, de la tapette. Elle part de la resignification de l'insulte qu'est le mot queer pour en faire un étendard théorique et politique pour repenser la sexualité, l'identité ou le genre à partir d'un développement de la réflexion sur le langage, le pouvoir et les corps telle que Foucault l'avait formulée dans le courant des années 70. Aujourd'hui, avec un retard de presque vingt ans, les publications queer fleurissent enfin dans le champ de la francophonie. Pour une introduction générale à cette pensée, on pourra lire, entre autres, la revue « Rue Descartes ", n40, 2003. Au côté des réflexions de De Lauretis, il nous faut au moins citer l'autre livre clé de ce mouvement de pensée :J. Butler, Trouble dans le genre. Pour un féminisme de la subversion, Paris, La découverte, 2005. Pour un historique des passages et de la réception de la philosophie française aux USA, on lira F. Cusset, French theory, Foucault, Derrida, Deleuze \& Cie et les mutations de la vie intellectuelle aux États-Unis, Paris, La découverte, 2003. En français, on retrouve quelques-uns des articles De Lauretis qui ont fondé la théorie queer dans T. 
De Lauretis, Théorie queer et cultures populaires. De Foucault à Cronenberg, La dispute, Coll. Le genre du monde, Paris, 2007.

6. Par exemple, dans la bibliographie que François Dosse consacre à Deleuze et Guattari, le nom de Pasolini n'apparaît qu'une seule fois et au sujet de l'approche qu'en donne Deleuze dans ses ouvrages consacrés au cinéma. Cf. F. Dosse, Gilles Deleuze et Félix Guattari., Op.cit. Le dernier livre de De Lauretis, mentionne de nombreuses références cinématographiques et explique le parcours qui l'a amenée à s'intéresser au cinéma mais à ne pas privilégier particulièrement le cinéma d'auteur cf. T. De Lauretis, Pulsions Freudiennes, Psychanalyse, Littérature et cinéma, P.U.F. Coll. Pratiques théoriques, 2010.

7. T. De Lauretis, Théorie queer et cultures populaires, Op. cit., p. 75.

8. M. Foucault, Histoire de la sexualité.Vol.1. La volonté de savoir, Paris, Gallimard, 1976.

9. F. Guattari, L'inconscient machinique. Essais de schizo-analyse, Fontenay-sous-bois, Recherches, coll. Encre, 1979, p. 75.

10. Ibid., p. 77.

11. Guattari, La révolution moléculaire, Op. cit., p. 218.

12. Ibid., p. 236.

13. Ibid., p. 236.

14. De lauretis, Théorie queer et cultures populaires, Op. cit., p. 75.

15. Ibid., p. 75.

16. On trouve en effet une analyse détaillée des films de Cronenberg dans les deux ouvrages déjà cités - de De Lauretis traduits en français.

17. C'est dans son Empirisme hérétique qu'il développe cette notion directement issue de la littérature. Il s'en sert pour indiquer la liberté qu'il utilise dans le cadrage de ses personnages: pour lui, le récit filmique ne doit pas assigner la parole ou l'image de façon précise à celui qui la prend ou à celui qui est en train de faire quelque chose mais peut rapporter les propos jusqu'à provoquer des sauts soudains, des changements d'orientation, des courants ou des flux de sens plus qu'une quelconque rationalité. Cf. P. P Pasolini., Empirismo eretico, Milano, Garzanti, 1972 (Traduction fr. L'Expérience hérétique : langue et cinéma, Paris, Payot, 1976).

18. J. Aumont, Du visage au cinéma, Paris, Cahiers du Cinéma, 1992, p. 60.

19. $\mathrm{Cf}$. Le très beau travail de découpage en image et de mise en séquence signifiante par thématique des différents films de Pasolini réalisé par M. Mancini et G. Perella (a cura di), Pier Paolo pasolini, Corpi e luoghi, Roma, Teorema,1981.

20. Si l'on en croit les dates de publications des articles, Foucault semble avoir avoir vu les films dans l'ordre inverse de leur sortie sur les écrans italien. Il commente donc d'abord Salo et, ensuite, Comizi d'amore. Les deux films étant respectivement recensés dans Cinématographe $\mathrm{n}^{\circ} 16$ en 1975 et dans Le Monde du 23 mars 1977. Ils sont repris aujourd'hui dans les M. Foucault, Dits et écrits, Paris, Gallimard Coll. Quarto, 2001, Vol. I (texte n²164) et Vol. II. (texte, 201).

21. Il reproche au film ce que l'intelligentsia de l'époque a globalement reproché à Pasolini : l'impossibilité de porter l'écriture sadienne à l'écran et le parti pris erroné de l'adaptation de l'action dans le contexte fasciste de la République de Salo. Dans le même sens cf. aussi le texte de R. Barthes, Pasolini-Sade, in « Le Monde » du 16 juin 1976.

22. M. Foucault, La volonté de savoir, Op. cit., p. 200.

23. Le bio-pouvoir étant le concept clé de Foucault pour résumer la façon dont le pouvoir s'exprime et se greffe à travers nos corps pour les rendre dociles et pour en façonner la vie.

24. On emploie ici "para» au sens de protection contre la vulgarité et l'homologation télévisuelle. D'ailleurs, comment pourrait-il en être autrement vu les positions de Pasolini quant au petit écran? cf. P. P. Pasolini, Contre la télévision et autres textes sur la politique et la société, Besançon, Les solitaires intempestifs, 2003.

25. C'est la traduction littérale de « comizi d'amore » qui est le titre original du film en italien. 
26. Pour plus de détails sur la question de l'abjuration, nous renvoyons au très beau livre de René Schérer qui développe un chapitre entier à ce propos en démontrant que l'abjuration de la Trilogie de la vie n'est que l'une des trois formes que peut prendre l'abjuration chez Pasolini. Cf. R. Schérer et G. Passerone, Passages pasoliniens, Presses Universitaires du Septentrion, Coll. Lettres et civilisations italienne, 2007.

27. Cf. P. Sollers, L'Ecriture et l'expérience des limites, Seuil, Paris, 1968 et A. Lebrun, Soudain un bloc d'abîme, Sade, Paris, Gallimard, 1986. Bien que Lebrun critique sévèrement les "badinages » intellectuels de Sollers, ils expriment tous deux la même position par rapport à la démarche de Sade, en particulier dans les 120 Journées de Sodome : il lui faut tout raconter. Le passage du roman qui explicite le plus le but de Sade est sans doute le suivant: «Sans doute beaucoup de tous les écarts que tu vas voir peints te déplairont, on le sait, mais il s'en trouvera quelques-uns qui t'échaufferont au point de te coûter du foutre, et voilà ce qu'il nous faut. Si nous n'avions pas tout dit, tout analysé comment voudrais-tu que nous eussions pu deviner ce qui te convient?". cf. Le Marquis de Sade, Les 120 journées de Sodome ou l'Ecole du libertinage, [1785], Paris, 10/18, 1975, p.74. Barthes aussi dans sa très belle lecture de Sade présente le Divin marquis comme un "logothète" à savoir: comme un inventeur d'une langue du texte qui ne viserait plus la communication mais l'impossibilité de se confronter à un ineffable. Cf. R. Barthes, Sade, Fourrier, Loyola, Paris, Seuil, 1971. Comme nous le disions plus haut, il avait également jugé sévèrement le film pour cette impossibilité de porter à l'écran ce genre de littérature. Sur ce point cf. l'excellent article de A. Naze, De silling à Salo. Usages pasoliniens de Sade in «Lignes : Penser Sade », 2004, n¹4, p. 107.

28. Ces girons font plus penser à l'architecture de Dante qu'à celle de Sade. C'est en tous cas la belle hypothèse de $\mathrm{H}$. Joubert-Laurencin, Portait du poète en cinéaste, Ecrits sur Pasolini, Besançon, Les solitaires Intempestifs, 2005.

29. C'est René Schérer qui dans son beau chapitre sur Salo avoue préférer - de manière absolument deleuzienne - le terme allégorie à celui de métaphore qu'emploie systématiquement Pasolini quand il s'exprime sur le sens de son film. Cf. R. Schérer et G. Passerone, Passages pasoliniens, Op. Cit.

30. Nous tirons cette citations issue du conférence dans un Lycée de Lecce du livre de Schérer, elle est issue de P. P. Pasolini, Opere Complete, Saggi sulla letteratura e sull'arte, Milano, Mondadori, coll. Meridiano, 1999, p.2858.

31. Cf. Amaury Voslion, Enfants de Salo. Entretiens avec quatre cinéastes Claire Denis, Bertrand Bonello, Gaspar Noé et Catherine Breillat, «bonus » du Dvd P. P. Pasolini, Salo ou les 120 Journées de Sodome (1975), Carlotta films prod., 2002.

32. Jean-Paul Curnier, La disparition des lucioles, in "Lignes: Pier Paolo Pasolini ", 2005, $\mathrm{n}^{\circ} 18$, p. 78-79.

33. Séquence sur laquelle Stéphane Nadaud a déjà brillamment attiré l'attention dans in $\mathrm{S}$. Nadaud, La ronde in «Lignes : Penser Sade », Op. Cit.

34. S. Murri, Pier Paolo Pasolini. Salo o le 120 giornate di Sodoma, Torino, Lindau, 2001, p. 10 (nous traduisons).

35. G. Rubin, Penser le sexe, in J. Butler-G.Rubin, Marché au sexe, E.P.E.L., Paris, 2001, p. 66.

36. Notre titre s'efforce de rendre hommage au beau livre de J. Butler, Défaire le genre, Paris, Amsterdam, 2006.

37. P. P. Pasolini, Scritti Corsari, Op. Cit., (Tr. fr. p. 146). P. P. Pasolini, Scritti corsari (1975), Garzanti, 1990, p. 126 (Trad. fr. Ecrits corsaires, Paris, Flammarion, 1976, p. 146.)

38. Ibid., p. 259 (Tr. fr. p. 256).

39. S. Daney, Persévérance. Entretiens avec Serge Toubiana, Paris, P.O.L., 1994, p. 85. 


\section{RÉSUMÉS}

L'article s'intéresse à la place qu'occupent les corps dans le cinéma de Pier Paolo Pasolini. Il analyse plus particulièrement deux films : d'une part, Commizi d'amore (1965), un documentaire pour la télévision italienne où le cinéaste questionne les mœurs et les représentations de ses compatriotes en matière de sexualité et, d'autre part, un point aveugle de l'histoire du cinéma, Salo ou les 120 journées de Sodome. On y détaille l'évolution de l'œuvre du cinéaste qui part d'un regard amusé sur ses contemporains pour devenir, peu à peu, noir et d'une rare violence. A l'aide de références théoriques qui vont de Guattari, à Foucault en passant par la théorie queer, l'article thématise l'importance de cette évolution non seulement d'un point de vue diégétique mais aussi du point de vue du « champ des visibilités ». Par cette expression, on essaye de cerner la manière dont à partir de ce qu'il est possible, ou pas, de voir dans une image cinématographique, le cinéma s'inscrit inconsciemment dans la construction et la normation de nos existences. Autrement dit, on y interroge le lien entre l'image pasolinienne, la sexualité et ses représentations. A lire la filmographie de Pasolini, on s'apercevra, en effet, que sa caméra problématise systématiquement le " genre » - au double sens du terme - pour tenter d'y semer le «trouble » : avec le cinéaste, les genres cinématographiques, tout comme le genre humain, sont remis en question dans l'évidence de leur certitude. Plus qu'insister sur les regrets pasoliniens face à la société contemporaine, l'article met donc en évidence son invitation constante à transgresser, par la poétique de l'image, toute normation du désir.

\section{AUTEUR}

\section{FABRICE BOURLEZ}

École Supérieure d'Art et de Design du Havre (Esah)

De nationalité belge, Fabrice Bourlez est docteur en philosophie (Université de Lille 3 et Università di Pisa). Il enseigne la philosophie et l'esthétique à l'Ecole Supérieure d'art et de Design de Reims (E.S.A.D.) et à l'Ecole Supérieure d'art du Havre (E.S.A.H). Sa thèse portait sur l'œuvre cinématographique de Pasolini. Il est l'auteur de différents articles sur le cinéma, la psychanalyse et les questions de "genre". Ses auteurs de prédilection sont Deleuze et Lacan. Il poursuit également une activité de traducteur de textes philosophiques (italien/français). Il collabore régulièrement pour le site web www.nonfiction.fr où il est coordinateur du pôle gender et féminisme. 